\title{
A family of anisotropic integral operators and behavior of its maximal eigenvalue
}

\author{
Boris S. Mityagin and Alexander V. Sobolev
}

Abstract. We study the family of compact integral operators $\mathbf{K}_{\beta}$ in $L^{2}(\mathbb{R})$ with the kernel

$$
K_{\beta}(x, y)=\frac{1}{\pi} \frac{1}{1+(x-y)^{2}+\beta^{2} \Theta(x, y)},
$$

depending on the parameter $\beta>0$, where $\Theta(x, y)$ is a symmetric non-negative homogeneous function of degree $\gamma \geq 1$. The main result is the following asymptotic formula for the maximal eigenvalue $M_{\beta}$ of $\mathbf{K}_{\beta}$ :

$$
M_{\beta}=1-\lambda_{1} \beta^{\frac{2}{\gamma+1}}+o\left(\beta^{\frac{2}{\gamma+1}}\right), \quad \beta \rightarrow 0,
$$

where $\lambda_{1}$ is the lowest eigenvalue of the operator $\mathbf{A}=|d / d x|+\Theta(x, x) / 2$. A central role in the proof is played by the fact that $\mathbf{K}_{\beta}, \beta>0$, is positivity improving. The case $\Theta(x, y)=\left(x^{2}+y^{2}\right)^{2}$ has been studied earlier in the literature as a simplified model of high-temperature superconductivity.

Mathematics Subject Classification (2010). Primary 45C05; Secondary 47A75.

Keywords. Eigenvalues, asymptotics, positivity improving integral operators, pseudo-differential operators, superconductivity.

\section{Introduction and the main result}

1.1. Introduction. The object of the study is the following family of integral operators on $L^{2}(\mathbb{R})$ :

$$
\mathbf{K}_{\beta} u(x)=\int K_{\beta}(x, y) u(y) d y,
$$

(here and below we omit the domain of integration if it is the entire real line $\mathbb{R}$ ) with the kernel

$$
K_{\beta}(x, y)=\frac{1}{\pi} \frac{1}{1+(x-y)^{2}+\beta^{2} \Theta(x, y)},
$$


where $\beta>0$ is a small parameter, and the function $\Theta=\Theta(x, y)$ is a homogeneous non-negative function of $x$ and $y$ such that

$$
\Theta(t x, t y)=t^{\gamma} \Theta(x, y), \quad \gamma>0,
$$

for all $x, y \in \mathbb{R}$ and $t>0$, and the following conditions are satisfied:

$$
\begin{cases}c \leq \Theta(x, y) \leq C, & |x|^{2}+|y|^{2}=1, \\ \Theta(x, y)=\Theta(y, x), & x, y \in \mathbb{R} .\end{cases}
$$

By $C$ or $c$ (with or without indices) we denote various positive constants whose value is of no importance. The conditions (3) and (4) guarantee that the operator $\mathbf{K}_{\beta}$ is self-adjoint and compact.

Such an operator, with $\Theta(x, y)=\left(x^{2}+y^{2}\right)^{2}$ was suggested by P. Krotkov and A. Chubukov in [6] and [7] as a simplified model of high-temperature superconductivity. The analysis in [6] and [7] reduces to the asymptotics of the top eigenvalue $M_{\beta}$ of the operator $\mathbf{K}_{\beta}$ as $\beta \rightarrow 0$. Heuristics in [6] and [7] suggest that $M_{\beta}$ should behave as $1-w \beta^{\frac{2}{5}}+o\left(\beta^{\frac{2}{5}}\right)$ with some positive constant $w$. A mathematically rigorous argument given by B. S. Mityagin in [9] produced a two-sided bound supporting this formula. The aim of the present paper is to find and justify an appropriate two-term asymptotic formula for $M_{\beta}$ as $\beta \rightarrow 0$ for a homogeneous function $\Theta$ satisfying (3), (4), and some additional smoothness conditions (see (8)).

As $\beta \rightarrow 0$, the operator $\mathbf{K}_{\beta}$ converges strongly to the positive-definite operator $\mathbf{K}_{0}$, which is no longer compact. The norm of $\mathbf{K}_{0}$ is easily found using the Fourier transform

$$
\hat{f}(\xi)=\frac{1}{\sqrt{2 \pi}} \int e^{-i \xi x} f(x) d x,
$$

which is unitary on $L^{2}(\mathbb{R})$. Then one checks directly that

$$
\begin{aligned}
& \text { the Fourier transform of } m_{t}(x)=\frac{t}{\pi} \frac{1}{t^{2}+x^{2}}, \quad t>0, \\
& \text { equals } \hat{m}_{t}(\xi)=\frac{1}{\sqrt{2 \pi}} e^{-t|\xi|}
\end{aligned}
$$

and hence the operator $\mathbf{K}_{0}$ is unitarily equivalent to the multiplication by the function $e^{-|\xi|}$, which means that $\left\|\mathbf{K}_{0}\right\|=1$.

1.2. The main result. For the maximal eigenvalue $M_{\beta}$ of the operator $\mathbf{K}_{\beta}$ denote by $\Psi_{\beta}$ a corresponding normalized eigenfunction. Note that the operator $\mathbf{K}_{\beta}$ is positivity improving, i.e for any non-negative non-zero function $u$ the function $\mathbf{K}_{\beta} u$ is positive a.a. $x \in \mathbb{R}$ (see [12], Chapter XIII.12). Thus, by [12], Theorem XIII.43 (or by [3], Theorem 13.3.6), the eigenvalue $M_{\beta}$ is non-degenerate and the eigenfunction $\Psi_{\beta}$ can be assumed to be positive a.a. $x \in \mathbb{R}$. From now on we always choose $\Psi_{\beta}$ in this 
way. Note in passing that due to the continuity of the kernel $K_{\beta}(x, y)$ in the variable $x$ the function $\Psi_{\beta}$ is in fact continuous and strictly positive for all $x \in \mathbb{R}$.

The behavior of $M_{\beta}$ as $\beta \rightarrow 0$, is governed by the model operator

$$
(\mathbf{A} u)(x)=\left|D_{x}\right| u(x)+2^{-1} \theta(x) u(x),
$$

where

$$
\theta(x)=\Theta(x, x)= \begin{cases}|x|^{\gamma} \Theta(1,1), & x \geq 0 \\ |x|^{\gamma} \Theta(-1,-1), & x<0 .\end{cases}
$$

This operator is understood as the pseudo-differential operator $\mathrm{Op}(a)$ with the symbol

$$
a(x, \xi)=|\xi|+2^{-1} \theta(x)
$$

For the sake of completeness recall that $P=\mathrm{Op}(p)$ is a pseudo-differential operator with the symbol $p=p(x, \xi)$ if

$$
(P u)(x)=\frac{1}{2 \pi} \iint e^{i(x-y) \xi} p(x, \xi) u(y) d y d \xi
$$

for any Schwartz class function $u$. The operator $\mathbf{A}$ is essentially self-adjoint on $C_{0}^{\infty}(\mathbb{R})$, and has a purely discrete spectrum (see e.g. [14], Theorems 26.2, 26.3). Using the von Neumann Theorem (see e.g. [11], Theorem X.25), one can see that $\mathbf{A}$ is self-adjoint on $D(\mathbf{A})=D\left(\left|D_{x}\right|\right) \cap D\left(|x|^{\gamma}\right)$, i.e $D(\mathbf{A})=H^{1}(\mathbb{R}) \cap L^{2}\left(\mathbb{R},|x|^{2 \gamma}\right)$. Denote by $\lambda_{l}>0, l=1,2, \ldots$ the eigenvalues of $\mathbf{A}$ arranged in ascending order, and by $\varphi_{l}$ the corresponding normalized eigenfunctions. As shown in Lemma 2, the lowest eigenvalue $\lambda_{1}$ is non-degenerate and its eigenfunction $\varphi_{1}$ can be chosen to be non-negative a.a. $x \in \mathbb{R}$. From now on we always choose $\varphi_{1}$ in this way.

The main result of this paper is contained in the next theorem.

Theorem 1. Let $\mathbf{K}_{\beta}$ be an integral operator defined by (1) with $\gamma \geq 1$. Suppose that the function $\Theta$ satisfies conditions (3), (4), and the following Lipshitz conditions:

$$
\begin{cases}|\Theta(t, 1)-\Theta(1,1)| \leq C|t-1|, & t \in(1-\varepsilon, 1+\varepsilon), \\ |\Theta(t,-1)-\Theta(-1,-1)| \leq C|t+1|, & t \in(-1-\varepsilon,-1+\varepsilon),\end{cases}
$$

with some $\varepsilon>0$. Let $M_{\beta}$ be the largest eigenvalue of the operator $\mathbf{K}_{\beta}$ and let $\Psi_{\beta}$ be the corresponding eigenfunction. Then

$$
\lim _{\beta \rightarrow 0} \beta^{-\frac{2}{\gamma+1}}\left(1-M_{\beta}\right)=\lambda_{1} .
$$

Moreover, the rescaled eigenfunctions $\alpha^{-\frac{1}{2}} \Psi_{\beta}\left(\alpha^{-1} \cdot\right), \alpha=\beta^{\frac{2}{\nu+1}}$, converge in norm to $\varphi_{1}$ as $\beta \rightarrow 0$. 
The eigenvalue $M_{\beta}$ was studied by B. Mityagin in [9] for $\Theta(x, y)=\left(x^{2}+y^{2}\right)^{\sigma}$, $\sigma>0$. It was conjectured that $\lim _{\beta \rightarrow 0} \beta^{-\frac{2}{2 \sigma+1}}\left(1-M_{\beta}\right)=L$ with some $L>0$, but only the two-sided bound

$$
c \beta^{\frac{2}{2 \sigma+1}} \leq 1-M_{\beta} \leq C \beta^{\frac{2}{2 \sigma+1}}
$$

with some constants $0<c \leq C$ was proved. It was also conjectured that in the case $\sigma=2$ the constant $L$ should coincide with the lowest eigenvalue of the operator $\left|D_{x}\right|+4 x^{4}$. Note that for this case the corresponding operator (6) is in fact $\left|D_{x}\right|+2 x^{4}$. J. Adduci found an approximate numerical value $\lambda_{1}=0.978 \ldots$ in this case, see [1].

Similar eigenvalue asymptotics were investigated by $\mathrm{H}$. Widom in [15] for integral operators with difference kernels. Some ideas of [15] are used in the proof of Theorem 1.

Let us now establish the non-degeneracy of the eigenvalue $\lambda_{1}$.

Lemma 2. Let $\mathbf{A}$ be as defined in (6). Then

(1) the semigroup $e^{-t \mathbf{A}}$ is positivity improving for all $t>0$,

(2) the lowest eigenvalue $\lambda_{1}$ is non-degenerate, and the corresponding eigenfunction $\varphi_{1}$ can be chosen to be positive a.a. $x \in \mathbb{R}$.

Proof. The non-degeneracy of $\lambda_{1}$ and positivity of the eigenfunction $\varphi_{1}$ would follow from the fact that $e^{-t \mathbf{A}}$ is positivity improving for all $t>0$, see [12], Theorem XIII.44. The proof of this fact is done by comparing the semigroups for the operators $\mathbf{A}$ and $\mathbf{A}_{0}=\left|D_{x}\right|$. Using (5) it is straightforward to find the integral kernel of $e^{-t \mathbf{A}_{0}}$ :

$$
m_{t}(x-y)=\frac{1}{\pi} \frac{t}{t^{2}+(x-y)^{2}}, \quad t>0,
$$

which shows that $e^{-t \mathbf{A}_{0}}$ is positivity improving. To extend the same conclusion to $e^{-t \mathbf{A}}$ let

$$
V_{n}(x)=\left\{\begin{array}{ll}
2^{-1} \theta(x), & |x| \leq n, \\
2^{-1} \theta( \pm n), & \pm x>n,
\end{array} \quad n=1,2, \ldots .\right.
$$

Since $\left(\mathbf{A}_{0}+V_{n}\right) f \rightarrow \mathbf{A} f$ and $\left(\mathbf{A}-V_{n}\right) f \rightarrow \mathbf{A}_{0} f$ as $n \rightarrow \infty$ for any $f \in C_{0}^{\infty}(\mathbb{R})$, by [10], Theorem VIII.25a, the operators $\mathbf{A}_{0}+V_{n}$ and $\mathbf{A}-V_{n}$ converge to $\mathbf{A}$ and $\mathbf{A}_{0}$ resp. in the strong resolvent sense as $n \rightarrow \infty$. Thus by [12], Theorem XIII.45, the semigroup $e^{-t \mathbf{A}}$ is also positivity improving for all $t>0$, as required.

1.3. Rescaling. As a rule, instead of $\mathbf{K}_{\beta}$ it is more convenient to work with the operator obtained by rescaling $x \rightarrow \alpha^{-1} x$ with $\alpha>0$. Precisely, let $U_{\alpha}$ be the unitary operator on $L^{2}(\mathbb{R})$ defined as $\left(U_{\alpha} f\right)(x)=\alpha^{-\frac{1}{2}} f\left(\alpha^{-1} x\right)$. Then $U_{\alpha} \mathbf{K}_{\beta} U_{\alpha}^{*}$ is the integral operator with the kernel

$$
\frac{\alpha}{\pi} \frac{1}{\alpha^{2}+(x-y)^{2}+\beta^{2} \alpha^{-\gamma+2} \Theta(x, y)} .
$$


Under the assumption $\beta^{2}=\alpha^{\gamma+1}$, this kernel becomes

$$
B_{\alpha}(x, y)=\frac{\alpha}{\pi} \frac{1}{\alpha^{2}+(x-y)^{2}+\alpha^{3} \Theta(x, y)} .
$$

Thus, denoting the corresponding integral operator by $\mathbf{B}_{\alpha}$, we get

$$
\mathbf{K}_{\beta}=U_{\alpha}^{*} \mathbf{B}_{\alpha} U_{\alpha}, \quad \alpha=\beta^{\frac{2}{\nu+\mathrm{T}}} .
$$

Henceforth the value of $\alpha$ is always chosen as in this formula.

Denote by $\mu_{\alpha}$ the maximal eigenvalue of the operator $\mathbf{B}_{\alpha}$, and by $\psi_{\alpha}$ - the corresponding normalized eigenfunction. By the same token as for the operator $\mathbf{K}_{\beta}$, the eigenvalue $\mu_{\alpha}$ is non-degenerate and the choice of the corresponding eigenfunction $\psi_{\alpha}$ is determined uniquely by the requirement that $\psi_{\alpha}>0$. Moreover,

$$
\mu_{\alpha}=M_{\beta}, \quad \psi_{\alpha}(x)=\left(U_{\alpha} \Psi_{\beta}\right)(x)=\alpha^{-\frac{1}{2}} \Psi_{\beta}\left(\alpha^{-1} x\right), \quad \alpha=\beta^{\frac{2}{\gamma+1}} .
$$

This rescaling allows one to rewrite Theorem 1 in a somewhat more compact form.

Theorem 3. Let $\gamma \geq 1$ and suppose that the function $\Theta$ satisfies conditions (3), (4), and (8). Then

$$
\lim _{\alpha \rightarrow 0} \alpha^{-1}\left(1-\mu_{\alpha}\right)=\lambda_{1} .
$$

Moreover, the eigenfunctions $\psi_{\alpha}$, converge in norm to $\varphi_{1}$ as $\alpha \rightarrow 0$.

The rest of the paper is devoted to the proof of Theorem 3, which immediately implies Theorem 1.

\section{2. "De-symmetrization" of $K_{\beta}$ and $B_{\alpha}$}

First we de-symmetrize the operator $\mathbf{K}_{\beta}$. Denote

$$
\mathbf{K}_{\beta}^{(l)} u(x)=\int K_{\beta}^{(l)}(x, y) u(y) d y,
$$

with the kernel

$$
K_{\beta}^{(l)}(x, y)=\frac{1}{\pi} \frac{1}{1+(x-y)^{2}+\beta^{2} \theta(x)} .
$$

Lemma 4. Let $\beta \leq 1$ and $\gamma \geq 1$. Suppose that the conditions (3), (4), and (8) are satisfied. Then

$$
\left\|\mathbf{K}_{\beta}^{(l)}-\mathbf{K}_{\beta}\right\| \leq C_{\gamma} \beta^{\frac{2}{\gamma}} .
$$


Proof. Due to (3) and (4),

$$
c(|t|+1)^{\gamma} \leq \Theta(t, \pm 1) \leq C(|t|+1)^{\gamma}, \quad t \in \mathbb{R} .
$$

Also,

$$
\left\{\begin{array}{l}
|\Theta(t, 1)-\Theta(1,1)| \leq C(|t|+1)^{\gamma-1}|t-1|, \\
|\Theta(t,-1)-\Theta(-1,-1)| \leq C(|t|+1)^{\gamma-1}|t+1|,
\end{array}\right.
$$

for all $t \in \mathbb{R}$. Indeed, (8) leads to the first inequality (14) for $|t-1|<\varepsilon$. For $|t-1| \geq \varepsilon$ it follows from (13) that

$$
|\Theta(t, 1)-\Theta(1,1)| \leq C(|t|+1)^{\gamma} \leq C^{\prime} \varepsilon^{-1}(|t|+1)^{\gamma-1}|t-1|
$$

The second bound in (14) is checked similarly.

Now we can estimate the difference of the kernels

$$
\begin{aligned}
& K_{\beta}(x, y)-K_{\beta}^{(l)}(x, y) \\
& \quad=\frac{1}{\pi} \frac{\beta^{2}(\Theta(x, x)-\Theta(x, y))}{\left(1+(x-y)^{2}+\beta^{2} \Theta(x, y)\right)\left(1+(x-y)^{2}+\beta^{2} \Theta(x, x)\right)} .
\end{aligned}
$$

It follows from (14) with $t=y|x|^{-1}$ that

$$
|\Theta(x, x)-\Theta(y, x)| \leq C(|x|+|y|)^{\gamma-1}|x-y| .
$$

Substituting into (15), we get

$$
\left|K_{\beta}(x, y)-K_{\beta}^{(l)}(x, y)\right| \leq C \frac{|x-y|}{\left(1+(x-y)^{2}\right)^{2-\delta}} \frac{\beta^{2}(|x|+|y|)^{\gamma-1}}{\left(1+\beta^{2}(|x|+|y|)^{\gamma}\right)^{\delta}},
$$

for any $\delta \in(0,1)$. The second factor on the right-hand side does not exceed

$$
\beta^{\frac{2}{\gamma}} \max _{t \geq 0} \frac{t^{\gamma-1}}{\left(1+t^{\gamma}\right)^{\delta}}=C \beta^{\frac{2}{\gamma}}
$$

under the assumption that $\delta \geq 1-\gamma^{-1}$. Therefore

$$
\left|K_{\beta}(x, y)-K_{\beta}^{(l)}(x, y)\right| \leq C \beta^{\frac{2}{\gamma}} \frac{|x-y|}{\left(1+(x-y)^{2}\right)^{2-\delta}} .
$$

For any $\delta \in(0,1)$ the right hand side is integrable in $x$ (or $y$ ). Now, estimating the norm using the standard Schur Test, see Proposition 15, we conclude that

$$
\left\|\mathbf{K}_{\beta}-\mathbf{K}_{\beta}^{(l)}\right\| \leq C \beta^{\frac{2}{\gamma}} \int \frac{|t|}{\left(1+t^{2}\right)^{2-\delta}} d t \leq C^{\prime} \beta^{\frac{2}{\gamma}},
$$

which is the required bound. 
Similarly to the operator $\mathbf{K}_{\beta}$, it is readily checked by scaling that the operator $\mathbf{K}_{\beta}^{(l)}$ is unitarily equivalent to the operator $\mathbf{B}_{\alpha}^{(l)}$ with the kernel

$$
B_{\alpha}^{(l)}(x, y)=\frac{1}{\pi} \frac{\alpha}{\alpha^{2}+(x-y)^{2}+\alpha^{3} \theta(x)} .
$$

Thus the bound (12) ensures that

$$
\left\|\mathbf{B}_{\alpha}-\mathbf{B}_{\alpha}^{(l)}\right\|=\left\|\mathbf{K}_{\beta}-\mathbf{K}_{\beta}^{(l)}\right\| \leq C \alpha^{1+\frac{1}{\gamma}}, \quad \alpha \leq 1,
$$

see (10) for the definition of $\alpha$.

\section{Approximation for $\mathbf{B}_{\alpha}^{(l)}$}

3.1. Symbol of $\mathbf{B}_{\boldsymbol{\alpha}}^{(l)}$. Now our aim is to show that the operator $I-\alpha \mathbf{A}$ is an approximation of the operator $\mathbf{B}_{\alpha}^{(l)}$, defined above. To this end we need to represent $\mathbf{B}_{\alpha}^{(l)}$ as a pseudo-differential operator. Rewriting the kernel (16) as

$$
B_{\alpha}^{(l)}(x, y)=t^{-1} m_{\alpha t}(x-y), \quad t=g_{\alpha}(x),
$$

with

$$
g_{\alpha}(x)=\sqrt{1+\alpha \theta(x)},
$$

and using (5), we can write for any Schwartz class function $u$ :

$$
\left(\mathbf{B}_{\alpha}^{(l)} u\right)(x)=\frac{1}{2 \pi} \iint e^{i(x-y) \xi} b_{\alpha}^{(l)}(x, \xi) u(y) d y d \xi
$$

where

$$
b_{\alpha}^{(l)}(x, \xi)=\frac{1}{g_{\alpha}(x)} e^{-\alpha|\xi| g_{\alpha}(x)}
$$

Thus $\mathbf{B}_{\alpha}^{(l)}=\operatorname{Op}\left(b_{\alpha}^{(l)}\right)$.

3.2. Approximation for $\mathbf{B}_{\boldsymbol{\alpha}}^{(l)}$. Let the operator $\mathbf{A}$ and the symbol $a(x, \xi)$ be as defined in (6) and (7). Our first objective is to check that the error

$$
r_{\alpha}(x, \xi)=b_{\alpha}^{(l)}(x, \xi)-(1-\alpha a(x, \xi))
$$

is small in a certain sense. The condition $\gamma \geq 1$ will allow us to use standard norm estimates for pseudo-differential operators. Using the formula

$$
e^{-\alpha y}=1-\alpha y+\alpha \int_{0}^{y}\left(1-e^{-\alpha t}\right) d t, \quad y>0,
$$


we can split the error as follows:

$$
\begin{gathered}
r_{\alpha}(x, \xi)=r_{\alpha}^{(1)}(x)+r_{\alpha}^{(2)}(x, \xi), \\
r_{\alpha}^{(1)}(x)=\frac{1}{g(x)}+\alpha 2^{-1} \theta(x)-1, \\
r_{\alpha}^{(2)}(x, \xi)=\frac{\alpha}{g(x)} \int_{0}^{|\xi| g(x)}\left(1-e^{-\alpha t}\right) d t,
\end{gathered}
$$

where we have used the notation $g(x)=g_{\alpha}(x)$ with $g_{\alpha}$ defined in (18). Since $\gamma \geq 1$, we have

$$
\left|g^{\prime}(x)\right| \leq C g(x), \quad C=C(\gamma), \quad x \neq 0,
$$

for all $\alpha \leq 1$. Introduce also the function $\zeta \in C^{\infty}\left(\mathbb{R}_{+}\right)$such that

$$
\zeta^{\prime}(x) \geq 0, \zeta(x)= \begin{cases}x, & 0 \leq x \leq 1 ; \\ 2, & x \geq 2\end{cases}
$$

Note that

$$
\zeta\left(x_{1} x_{2}\right) \leq 2 \zeta\left(x_{1}\right) x_{2}, \quad x_{1} \geq 0, x_{2} \geq 1 .
$$

We study the above components $r^{(1)}, r^{(2)}$ separately and introduce the function

$$
e_{\alpha}^{(1)}(x)=\frac{1}{\langle x\rangle^{\gamma} \zeta\left(\alpha\langle x\rangle^{\gamma}\right)} r_{\alpha}^{(1)}(x)
$$

and the symbol

$$
e_{\alpha}^{(2)}(x, \xi)=g_{\alpha}(x)^{-\varkappa}\left(\zeta((\alpha\langle\xi\rangle))^{\varkappa}\langle\xi\rangle\right)^{-1} r_{\alpha}^{(2)}(x, \xi),
$$

where $x \in(0,1]$ is a fixed number. To avoid cumbersome notation the dependence of $e_{\alpha}^{(2)}$ on $\varkappa$ is not reflected in the notation. We denote the operators $\operatorname{Op}\left(r_{\alpha}\right)$ and $\operatorname{Op}\left(e_{\alpha}\right)$ by $\mathbf{R}_{\alpha}$ and $\mathbf{E}_{\alpha}$ respectively (with or without superscripts).

Lemma 5. Let $\gamma \geq 1$. Then for all $\alpha>0$,

$$
\left\|e_{\alpha}^{(1)}\right\|_{L^{\infty}} \leq C \alpha .
$$

Proof. Estimate the function $r_{\alpha}^{(1)}$ :

$$
\left|r_{\alpha}^{(1)}(x)\right| \leq \begin{cases}C \alpha^{2}|x|^{2 \gamma}, & \alpha \theta(x) \leq 1 / 2, \\ C \alpha|x|^{\gamma}, & \alpha \theta(x)>1 / 2,\end{cases}
$$

with a constant $C$ independent of $x$. The second estimate is immediate, and the first one follows from the Taylor's formula

$$
\frac{1}{\sqrt{1+t}}=1-\frac{t}{2}+O\left(t^{2}\right), \quad 0 \leq t \leq \frac{1}{2} .
$$


Thus

$$
\left|r_{\alpha}^{(1)}(x)\right| \leq C \alpha|x|^{\gamma} \zeta\left(\alpha|x|^{\gamma}\right) .
$$

This leads to the proclaimed estimate for $e_{\alpha}^{(1)}$.

Lemma 6. Let $\gamma \geq 1$. Then for all $\alpha>0$ and any $\varkappa \in(0,1]$,

$$
\left\|\mathbf{E}_{\alpha}^{(2)}\right\| \leq C_{\varkappa} \alpha \text {. }
$$

Proof. To estimate the norm of $\left.\mathrm{Op}\left(e_{\alpha}^{(2)}\right)\right)$ we use Proposition 16. It is clear that the distributional derivatives $\partial_{x}, \partial_{\xi}, \partial_{x} \partial_{\xi}$ of the symbol $e_{\alpha}^{(2)}(x, \xi)$ exist and are given by

$$
\begin{aligned}
\partial_{x} r_{\alpha}^{(2)}(x, \xi) & =-\frac{\alpha}{g^{2}} g^{\prime} \int_{0}^{|\xi| g}\left(1-e^{-\alpha t}\right) d t+\frac{\alpha}{g}|\xi| g^{\prime}\left(1-e^{-\alpha|\xi| g}\right), \\
\partial_{\xi} r_{\alpha}^{(2)}(x, \xi) & =\alpha \operatorname{sign} \xi\left(1-e^{-\alpha|\xi| g}\right), \\
\partial_{x} \partial_{\xi} r_{\alpha}^{(2)}(x, \xi) & =\alpha^{2} \xi g^{\prime} e^{-\alpha|\xi| g},
\end{aligned}
$$

for all $x \neq 0, \xi \neq 0$. For any $\varkappa \in(0,1]$ the elementary bounds hold:

$$
\begin{aligned}
\int_{0}^{|\xi| g}\left(1-e^{-\alpha t}\right) d t & \leq|\xi| g \zeta\left((\alpha|\xi| g)^{\varkappa}\right) \leq 2|\xi| g^{1+\varkappa} \zeta\left((\alpha|\xi|)^{\varkappa}\right), \\
\left|1-e^{-\alpha|\xi| g \mid}\right| & \leq \zeta\left((\alpha|\xi| g)^{\varkappa}\right) \leq 2 g^{\varkappa} \zeta\left((\alpha|\xi|)^{\varkappa}\right), \\
\alpha|\xi| g e^{-\alpha|\xi| g} & \leq \zeta\left((\alpha|\xi| g)^{\varkappa}\right) \leq 2 g^{\varkappa} \zeta\left((\alpha|\xi|)^{\varkappa}\right) .
\end{aligned}
$$

Here we have used (20). Thus, in view of (19),

$$
\left|r_{\alpha}^{(2)}(x, \xi)\right|+\left|\partial_{\xi} r_{\alpha}^{(2)}(x, \xi)\right|+\left|\partial_{x} r_{\alpha}^{(2)}(x, \xi)\right| \leq C \alpha\langle\xi\rangle g^{\varkappa} \zeta\left((\alpha|\xi|)^{\varkappa}\right) .
$$

Also,

$$
\left|\partial_{x} \partial_{\xi} r_{\alpha}^{(2)}(x, \xi)\right| \leq \alpha \frac{\left|g^{\prime}\right|}{g}\left(\alpha|\xi| g e^{-\alpha|\xi| g}\right) \leq C \alpha|g|^{\varkappa} \zeta\left((\alpha|\xi|)^{\varkappa}\right) .
$$

Now estimate the derivatives of the weights:

$$
\begin{array}{r}
\left|\partial_{x} g^{-\varkappa}\right|=\varkappa g^{-\varkappa-1} g^{\prime} \leq C g^{-\varkappa}, \quad x \neq 0, \\
\left|\partial_{\xi}\left(\langle\xi\rangle \zeta\left((\alpha\langle\xi\rangle)^{\varkappa}\right)\right)^{-1}\right| \leq C \frac{1}{\langle\xi\rangle^{2} \zeta\left((\alpha\langle\xi\rangle)^{\varkappa)}\right.}, \quad \xi \in \mathbb{R} .
\end{array}
$$

Thus the symbol $e_{\alpha}^{(2)}(x, \xi)$ as well as its derivatives $\partial_{x}, \partial_{\xi}, \partial_{x} \partial_{\xi}$ are bounded by $C \alpha$ for all $\alpha>0$ uniformly in $x, \xi$. Now the required estimate follows from Proposition 16.

We make a useful observation. 
Corollary 7. Let $\gamma \geq 1$ and $\varkappa \in(0,1]$. Then for any function $f \in D(\mathbf{A})$,

$$
\begin{gathered}
\alpha^{-1}\left\|\mathbf{R}_{\alpha}^{(1)} f\right\| \rightarrow 0, \quad \alpha \rightarrow 0, \\
\alpha^{-1}\left\|\mathbf{E}_{\alpha}^{(2)}\left\langle D_{x}\right\rangle \zeta\left(\left(\alpha\left\langle D_{x}\right\rangle\right)^{\varkappa}\right) f\right\| \rightarrow 0, \quad \alpha \rightarrow 0 .
\end{gathered}
$$

Proof. Rewrite:

$$
\left\|\mathbf{R}_{\alpha}^{(1)} f\right\|=\left\|\mathbf{E}_{\alpha}^{(1)}\langle x\rangle^{\gamma} \zeta\left(\alpha\langle x\rangle^{\gamma}\right) f\right\| \leq\left\|\mathbf{E}_{\alpha}^{(1)}\right\|\left\|\langle x\rangle^{\gamma} \zeta\left(\alpha\langle x\rangle^{\gamma}\right) f\right\| .
$$

By Lemma 5 the norm of $\mathbf{E}_{\alpha}^{(1)}$ on the right-hand side is bounded by $C \alpha$. The function $\langle x\rangle^{\gamma} \zeta\left(\alpha\langle x\rangle^{\gamma}\right) f$ tends to zero as $\alpha \rightarrow 0$ a.a. $x \in \mathbb{R}$, and it is uniformly bounded by the function $\langle x\rangle^{\gamma}|f|$, which belongs to $L^{2}$, since $f \in D(\mathbf{A})$. Thus the second factor in (25) tends to zero as $\alpha \rightarrow 0$ by the Dominated Convergence Theorem. This proves (23).

Proof of (24). Estimate:

$$
\left\|\mathbf{E}_{\alpha}^{(2)}\left\langle D_{x}\right\rangle \zeta\left(\left(\alpha\left\langle D_{x}\right\rangle\right)^{\varkappa}\right) f\right\| \leq\left\|\mathbf{E}_{\alpha}^{(2)}\right\|\left\|\langle\xi\rangle \zeta\left((\alpha\langle\xi\rangle)^{\varkappa}\right) \hat{f}\right\| .
$$

By Lemma 6 the norm of the first factor on the right-hand side is bounded by $C \alpha$. The second factor tends to zero as $\alpha \rightarrow 0$ for the same reason as in the proof of (23).

\section{Norm-convergence of the extremal eigenfunction}

Recall that the maximal positive eigenvalue $\mu_{\alpha}$ of the operator $\mathbf{B}_{\alpha}$ is non-degenerate, and the corresponding (normalized) eigenfunction $\psi_{\alpha}$ is positive a.a. $x \in \mathbb{R}$.

The principal goal of this section is to prove that any infinite subset of the family $\psi_{\alpha}, \alpha \leq 1$ contains a norm-convergent sequence. We begin with an upper bound for $1-\mu_{\alpha}$ which will be crucial for our argument.

Lemma 8. If $\gamma \geq 1$, then

$$
\limsup _{\alpha \rightarrow 0} \alpha^{-1}\left(1-\mu_{\alpha}\right) \leq \lambda_{1} .
$$

Proof. Denote $\varphi=\varphi_{1}$. By a straightforward variational argument it follows that

$$
\begin{aligned}
\mu_{\alpha} & \geq\left(\mathbf{B}_{\alpha} \varphi, \varphi\right) \geq\left|\left(\mathbf{B}_{\alpha}^{(l)} \varphi, \varphi\right)\right|-\left\|\mathbf{B}_{\alpha}-\mathbf{B}_{\alpha}^{(l)}\right\| \\
& \geq((I-\alpha \mathbf{A}) \varphi, \varphi)-\left|\left(\mathbf{R}_{\alpha} \varphi, \varphi\right)\right|+o(\alpha) \\
& =1-\alpha \lambda_{1}-\left|\left(\mathbf{R}_{\alpha} \varphi, \varphi\right)\right|+o(\alpha),
\end{aligned}
$$

where we have also used (17). By definitions (21) and (22),

$$
\left|\left(\mathbf{R}_{\alpha} \varphi, \varphi\right)\right| \leq\left\|\mathbf{R}_{\alpha}^{(1)} \varphi\right\|+\left\|\mathbf{E}_{\alpha}^{(2)}\left\langle D_{x}\right\rangle \zeta\left(\left(\alpha\left\langle D_{x}\right\rangle\right)^{\varkappa}\right) \varphi\right\|\left\|g_{\alpha}^{\varkappa} \varphi\right\|,
$$

where $\varkappa \in(0,1]$. It is clear that $g_{\alpha}^{\varkappa} \varphi \in L^{2}$ and its norm is bounded uniformly in $\alpha \leq 1$. The remaining terms on the right-hand side are $o(\alpha)$ due to Corollary 7. This leads to (26). 
The established upper bound leads to the following property.

Lemma 9. For any $x \in(0,1)$,

$$
\left\|g_{\alpha}^{\varkappa} \psi_{\alpha}\right\| \leq C
$$

uniformly in $\alpha \leq 1$.

Proof. By definition of $\psi_{\alpha}$,

$$
g_{\alpha}^{\varkappa} \psi_{\alpha}=\mu_{\alpha}^{-1} g_{\alpha}^{\varkappa} \mathbf{B}_{\alpha} \psi_{\alpha} .
$$

In view of (4), by definition (18) we have $\Theta(x, y) \geq C|x|^{\gamma} \geq c \theta(x)$, so that the kernel $B_{\alpha}(x, y)$ is bounded from above by

$$
B_{\alpha}(x, y) \leq \frac{\alpha}{\pi} \frac{C}{(x-y)^{2}+\alpha^{2} g_{\alpha}(x)^{2}},
$$

and thus the kernel $\widetilde{B}_{\alpha}(x, y)=g_{\alpha}(x)^{\varkappa} B_{\alpha}(x, y)$ satisfies the estimate

$$
\widetilde{B}_{\alpha}(x, y) \leq \frac{C}{\pi \alpha} \frac{1}{\left(1+\alpha^{-2}(x-y)^{2}\right)^{1-\frac{\alpha}{2}}} .
$$

Since $x<1$, by Proposition 15 this kernel defines a bounded operator with the norm uniformly bounded in $\alpha>0$. Thus

$$
\left\|g_{\alpha}^{\varkappa} \psi_{\alpha}\right\| \leq C \mu_{\alpha}^{-1}\left\|\psi_{\alpha}\right\| \leq C \mu_{\alpha}^{-1} .
$$

It remains to observe that by Lemma 8 the eigenvalue $\mu_{\alpha}$ is separated from zero uniformly in $\alpha \leq 1$.

Now we obtain more delicate estimates for $\psi_{\alpha}$. For a number $h \geq 0$ introduce the function

$$
S_{\alpha}(t ; h)=\frac{\alpha}{\pi} \frac{1}{\alpha^{2}+t^{2}+h}, \quad t \in \mathbb{R},
$$

and denote by $\mathbf{S}_{\alpha}(h)$ the integral operator with the kernel $\mathbf{S}_{\alpha}(x-y ; h)$. Along with $\mathbf{S}_{\alpha}(h)$ we also consider the operator

$$
\mathbf{T}_{\alpha}(h)=\mathbf{S}_{\alpha}(0)-\mathbf{S}_{\alpha}(h) .
$$

Due to (5) the Fourier transform of $S_{\alpha}(t ; h)$ is

$$
\widehat{S}_{\alpha}(\xi ; h)=\frac{\alpha}{\sqrt{2 \pi} \sqrt{\alpha^{2}+h}} e^{-|\xi| \sqrt{\alpha^{2}+h}}, \quad \xi \in \mathbb{R},
$$

so that

$$
\left\|\mathbf{S}_{\alpha}(h)\right\|=\frac{\alpha}{\sqrt{\alpha^{2}+h}}, \quad\left\|\mathbf{T}_{\alpha}(h)\right\|=1-\frac{\alpha}{\sqrt{\alpha^{2}+h}} .
$$

Denote by $\chi_{R}$ the characteristic function of the interval $(-R, R)$. 
Lemma 10. For sufficiently small $\alpha>0$ and $\alpha R \leq 1$,

$$
\left\|\hat{\psi}_{\alpha} \chi_{R}\right\|^{2} \geq 1-\frac{4 \lambda_{1}}{R} .
$$

Proof. Since $B_{\alpha}(x, y)<S_{\alpha}(x-y ; 0)$ (see (9) and (27)) and $\psi_{\alpha} \geq 0$, we can write, using (28):

$$
\begin{aligned}
\mu_{\alpha} & =\left(\mathbf{B}_{\alpha} \psi_{\alpha}, \psi_{\alpha}\right)<\int_{\mathbb{R}} \int_{\mathbb{R}} S_{\alpha}(x-y ; 0) \psi_{\alpha}(x) \psi_{\alpha}(y) d x d y=\int_{\mathbb{R}} e^{-\alpha|\xi|}\left|\hat{\psi}_{\alpha}(\xi)\right|^{2} d \xi \\
& \leq \int_{|\xi| \leq R}\left|\hat{\psi}_{\alpha}(\xi)\right|^{2} d \xi+e^{-\alpha R} \int_{|\xi|>R}\left|\hat{\psi}_{\alpha}(\xi)\right|^{2} d \xi \\
& =\left(1-e^{-\alpha R}\right) \int_{|\xi| \leq R}\left|\hat{\psi}_{\alpha}(\xi)\right|^{2} d \xi+e^{-\alpha R} .
\end{aligned}
$$

Due to (26), $\mu_{\alpha} \geq 1-2 \alpha \lambda_{1}$ for sufficiently small $\alpha$, so

$$
1-e^{-\alpha R}-2 \alpha \lambda_{1} \leq\left(1-e^{-\alpha R}\right)\left\|\hat{\psi}_{\alpha} \chi_{R}\right\|^{2},
$$

which implies that

$$
\left\|\hat{\psi}_{\alpha} \chi_{R}\right\|^{2} \geq 1-\frac{2 \alpha \lambda_{1}}{1-e^{-\alpha R}} .
$$

Since $e^{-s} \leq(1+s)^{-1}$ for all $s \geq 0$, we get $\left(1-e^{-s}\right)^{-1} \leq 2 s^{-1}$ for $0<s \leq 1$, which entails (30) for $\alpha R \leq 1$.

Lemma 11. For sufficiently small $\alpha>0$ and any $R>0$,

$$
\left\|\psi_{\alpha} \chi_{R}\right\| \geq 1-4 \alpha \lambda_{1}-\frac{C}{R^{\gamma}}
$$

with some constant $C>0$ independent of $\alpha$ and $R$.

Proof. It follows from (4) that $\Theta(x, y) \geq c|x|^{\gamma}$, so that the kernel $B_{\alpha}(x, y)$ satisfies the bound

$$
B_{\alpha}(x, y) \leq S_{\alpha}\left(x-y ; c \alpha^{3} R^{\gamma}\right), \quad \text { for }|x| \geq R>0 .
$$

Since $\psi_{\alpha} \geq 0$,

$$
\begin{aligned}
\mu_{\alpha} & =\left(\mathbf{B}_{\alpha} \psi_{\alpha}, \psi_{\alpha}\right) \leq\left(\mathbf{S}_{\alpha}(0) \psi_{\alpha}, \psi_{\alpha} \chi_{R}\right)+\left(\mathbf{S}_{\alpha}\left(c \alpha^{3} R^{\gamma}\right) \psi_{\alpha}, \psi_{\alpha}\left(1-\chi_{R}\right)\right) \\
& =\left(\mathbf{T}_{\alpha}\left(c \alpha^{3} R^{\gamma}\right) \psi_{\alpha}, \psi_{\alpha} \chi_{R}\right)+\left(\mathbf{S}_{\alpha}\left(c \alpha^{3} R^{\gamma}\right) \psi_{\alpha}, \psi_{\alpha}\right) .
\end{aligned}
$$

In view of (29),

$$
\begin{aligned}
\mu_{\alpha} & \leq\left\|\mathbf{T}_{\alpha}\left(c \alpha^{3} R^{\gamma}\right)\right\|\left\|\psi_{\alpha} \chi_{R}\right\|+\left\|\mathbf{S}_{\alpha}\left(c \alpha^{3} R^{\gamma}\right)\right\| \\
& =\left(1-\frac{1}{\sqrt{1+c \alpha R^{\gamma}}}\right)\left\|\psi_{\alpha} \chi_{R}\right\|+\frac{1}{\sqrt{1+c \alpha R^{\gamma}}} .
\end{aligned}
$$


Using, as in the proof of the previous lemma, the bound (26), we obtain that

$$
1-\frac{1}{\sqrt{1+c \alpha R^{\gamma}}}-2 \alpha \lambda_{1} \leq\left(1-\frac{1}{\sqrt{1+c \alpha R^{\gamma}}}\right)\left\|\psi_{\alpha} \chi_{R}\right\|,
$$

so

$$
1-\frac{4 \lambda_{1}\left(1+c \alpha R^{\gamma}\right)}{c R^{\gamma}} \leq\left\|\psi_{\alpha} \chi_{R}\right\|
$$

This entails (31).

Now we show that any sequence from the family $\psi_{\alpha}$ contains a norm-convergent subsequence. The proof is inspired by [15], Lemma 7. We precede it with the following elementary result.

Lemma 12. Let $f_{j} \in L^{2}(\mathbb{R})$ be a sequence such that $\left\|f_{j}\right\| \leq C$ uniformly in $j=1,2, \ldots$, and $f_{j}(x)=0$ for all $|x| \geq \rho>0$ and all $j=1,2, \ldots$. Suppose that $f_{j}$ converges weakly to $f \in L^{2}(\mathbb{R})$ as $j \rightarrow \infty$, and that for some constant $A>0$, and all $R \geq R_{0}>0$,

$$
\left\|\hat{f}_{j} \chi_{R}\right\| \geq A-C R^{-\delta}, \quad \delta>0,
$$

uniformly in $j$. Then $\|f\| \geq A$.

Proof. Since $f_{j}$ are uniformly compactly supported, the Fourier transforms $\hat{f}_{j}(\xi)$ converge to $\hat{f}(\xi)$ a.a. $\xi \in \mathbb{R}^{d}$ as $j \rightarrow \infty$. Moreover, the sequence $\hat{f}_{j}(\xi)$ is uniformly bounded, so $\hat{f}_{j} \chi_{R} \rightarrow \hat{f} \chi_{R}, j \rightarrow \infty$ in $L^{2}(\mathbb{R})$ for any $R>0$. Therefore (32) implies that

$$
\left\|\hat{f} \chi_{R}\right\| \geq A-C R^{-\delta} \text {. }
$$

Since $R$ is arbitrary, we have $\|f\|=\|\hat{f}\| \geq A$, as claimed.

Lemma 13. For any sequence $\alpha_{n} \rightarrow 0, n \rightarrow \infty$, there exists a subsequence $\alpha_{n_{k}} \rightarrow 0$, $k \rightarrow \infty$, such that the eigenfunctions $\psi_{\alpha_{n_{k}}}$ converge in norm as $k \rightarrow \infty$.

Proof. Since the functions $\psi_{\alpha}, \alpha \geq 0$ are normalized, there is a subsequence $\psi_{\alpha_{n_{k}}}$ which converges weakly. Denote the limit by $\psi$. From now on we write $\psi_{k}$ instead of $\psi_{\alpha_{n_{k}}}$ to avoid cumbersome notation. In view of the relations

$$
\left\|\psi_{k}-\psi\right\|^{2}=1+\|\psi\|^{2}-2 \operatorname{Re}\left(\psi_{k}, \psi\right) \rightarrow 1-\|\psi\|^{2}, \quad k \rightarrow \infty,
$$

it suffices to show that $\|\psi\|=1$.

Fix a number $\rho>0$, and split $\psi_{k}$ in the following way:

$$
\psi_{k}(x)=\psi_{k, \rho}^{(1)}(x)+\psi_{k, \rho}^{(2)}(x), \quad \psi_{k, \rho}^{(1)}(x)=\psi_{k}(x) \chi_{\rho}(x) .
$$


Clearly, $\psi_{k, \rho}^{(1)}$ converges weakly to $w_{\rho}=\psi \chi_{\rho}$ as $k \rightarrow \infty$. Assume that $\alpha_{n_{k}} \leq \rho^{-\gamma}$, so that by (31),

$$
\left\|\psi_{k, \rho}^{(1)}\right\|^{2} \geq 1-\frac{C}{\rho^{\gamma}}, \quad\left\|\psi_{k, \rho}^{(2)}\right\|^{2} \leq \frac{C}{\rho^{\gamma}} .
$$

Therefore, for any $R>0$,

$$
\left\|\widehat{\psi_{k, \rho}^{(1)}} \chi_{R}\right\| \geq\left\|\hat{\psi}_{k} \chi_{R}\right\|-\left\|\psi_{k, \rho}^{(2)}\right\| \geq 1-4 \lambda_{1} R^{-1}-C \rho^{-\frac{\gamma}{2}},
$$

where we have used (30). By Lemma 12,

$$
\left\|w_{\rho}\right\| \geq 1-C \rho^{-\frac{\gamma}{2}} .
$$

Since $\rho$ is arbitrary, $\|\psi\| \geq 1$, and hence $\|\psi\|=1$. As a result, the sequence $\psi_{k}$ converges in norm, as claimed.

\section{Asymptotics of $\mu_{\alpha}, \alpha \rightarrow 0$ : proof of Theorem 1}

As before, by $\lambda_{l}, l=1,2, \ldots$ we denote the eigenvalues of $\mathbf{A}$ arranged in ascending order, and by $\varphi_{l}$ the corresponding normalized eigenfunctions. Recall that the lowest eigenvalue $\lambda_{1}$ of the model operator $\mathbf{A}$ is non-degenerate and its (normalized) eigenfunction $\varphi_{1}$ is chosen to be positive a.a. $x \in \mathbb{R}$. We begin with proving Theorem 3.

Proof of Theorem 3. The proof essentially follows the plan of [15]. It suffices to show that for any sequence $\alpha_{n} \rightarrow 0, n \rightarrow \infty$, one can find a subsequence $\alpha_{n_{k}} \rightarrow 0$, $k \rightarrow \infty$ such that

$$
\lim _{k \rightarrow \infty} \alpha_{n_{k}}^{-1}\left(1-\mu_{\alpha_{n_{k}}}\right)=\lambda_{1},
$$

and $\psi_{\alpha_{n_{k}}}$ converges in norm to $\varphi_{1}$ as $k \rightarrow \infty$. By Lemma 13 one can pick a subsequence $\alpha_{n_{k}}$ such that $\psi_{\alpha_{n_{k}}}$ converges in norm as $k \rightarrow \infty$. As in the proof of Lemma 13 denote by $\psi$ the limit, so $\|\psi\|=1$ and $\psi \geq 0$ a.e. For simplicity we write $\psi_{\alpha}$ instead of $\psi_{\alpha_{n_{k}}}$. For an arbitrary function $f \in D(\mathbf{A})$ write

$$
\begin{aligned}
\mu_{\alpha}\left(\psi_{\alpha}, f\right) & =\left(\mathbf{B}_{\alpha} \psi_{\alpha}, f\right)=\left(\psi_{\alpha}, \mathbf{B}_{\alpha}^{(l)} f\right)+\left(\psi_{\alpha},\left(\mathbf{B}_{\alpha}-\mathbf{B}_{\alpha}^{(l)}\right) f\right) \\
& =\left(\psi_{\alpha}, f\right)-\alpha\left(\psi_{\alpha}, \mathbf{A} f\right)+\left(\psi_{\alpha}, \mathbf{R}_{\alpha} f\right)+\left(\psi_{\alpha},\left(\mathbf{B}_{\alpha}-\mathbf{B}_{\alpha}^{(l)}\right) f\right) .
\end{aligned}
$$

This implies that

$$
\alpha^{-1}\left(1-\mu_{\alpha}\right)\left(\psi_{\alpha}, f\right)=\left(\psi_{\alpha}, \mathbf{A} f\right)-\alpha^{-1}\left(\psi_{\alpha}, \mathbf{R}_{\alpha} f\right)-\alpha^{-1}\left(\psi_{\alpha},\left(\mathbf{B}_{\alpha}-\mathbf{B}_{\alpha}^{(l)}\right) f\right) \text {. }
$$

In view of (17) the last term on the right-hand side tends to zero as $\alpha \rightarrow 0$. The first term trivially tends to $(\psi, \mathbf{A} f)$. Consider the second term:

$$
\begin{aligned}
\left|\left(\psi_{\alpha}, \mathbf{R}_{\alpha} f\right)\right| & =\left(\psi_{\alpha}, \mathbf{R}_{\alpha}^{(1)} f\right)+\left(g_{\alpha}^{\varkappa} \psi_{\alpha}, \mathbf{E}_{\alpha}^{(2)}\left\langle D_{x}\right\rangle \zeta\left(\left(\alpha\left\langle D_{x}\right\rangle\right)^{\varkappa}\right) f\right) \\
& \leq\left\|\mathbf{R}_{\alpha}^{(1)} f\right\|+\left\|g_{\alpha}^{\varkappa} \psi_{\alpha}\right\|\left\|\mathbf{E}_{\alpha}^{(2)}\left\langle D_{x}\right\rangle \zeta\left(\left(\alpha\left\langle D_{x}\right\rangle\right)^{\varkappa}\right) f\right\| .
\end{aligned}
$$


Assume now that $\varkappa<1$. By Corollary 7 and Lemma 9, the right-hand side is $o(\alpha)$, and hence, if $(\psi, f) \neq 0$, then passing to the limit in (33) we get

$$
\lim _{\alpha \rightarrow 0} \alpha^{-1}\left(1-\mu_{\alpha}\right)=\frac{(\psi, \mathbf{A} f)}{(\psi, f)} .
$$

Let $f=\varphi_{l}$ with some $l$, so that $(\psi, \mathbf{A} f)=\lambda_{l}\left(\psi, \varphi_{l}\right)$. Suppose that $\left(\psi, \varphi_{l}\right) \neq 0$, so that

$$
\lim _{\alpha \rightarrow 0} \alpha^{-1}\left(1-\mu_{\alpha}\right)=\lambda_{l}
$$

By the uniqueness of the above limit, $\left(\psi, \varphi_{j}\right)=0$ for all $j$ 's such that $\lambda_{j} \neq \lambda_{k}$. Thus, by completeness of the system $\left\{\varphi_{k}\right\}$, the function $\psi$ is an eigenfunction of $\mathbf{A}$ with the eigenvalue $\lambda_{l}$. In view of (26), $\lambda_{l} \leq \lambda_{1}$. Since the eigenvalues $\lambda_{j}$ are labeled in ascending order we conclude that $\lambda_{l}=\lambda_{1}$. As this eigenvalue is non-degenerate and the corresponding eigenfunction $\varphi_{1}$ is positive a.e., we observe that $\psi=\varphi_{1}$.

Proof of Theorem 1. Theorem 1 follows from Theorem 3 due to the relations (11).

\section{Miscellaneous}

In this short section we collect some open questions related to the spectrum of the operator (1).

6.1. Theorems 1 and 3 give information on the largest eigenvalue $M_{\beta}$ of the operator $\mathbf{K}_{\beta}$ defined in (1), (2). Let

$$
M_{\beta} \equiv M_{1, \beta}>M_{2, \beta} \geq \ldots
$$

be the sequence of all positive eigenvalues of $\mathbf{K}_{\beta}$ arranged in descending order. The following conjecture is a natural extension of Theorem 1.

Conjecture 14. For any $j=1,2, \ldots$

$$
\lim _{\beta \rightarrow 0} \beta^{-\frac{2}{\gamma+1}}\left(1-M_{j, \beta}\right)=\lambda_{j},
$$

where $\lambda_{1}<\lambda_{2} \leq \ldots$ are eigenvalues of the operator $\mathbf{A}$ defined in (6), arranged in ascending order.

For the case $\Theta(x, y)=\left(x^{2}+y^{2}\right)^{2}$ the formula (35) was conjectured in [9], Section 7.1, but without specifying what the values $\lambda_{j}$ are. As in [9], the formula (35) is prompted by the paper [15] where asymptotics of the form (35) were found for an integral operator with a difference kernel. 
6.2. Although the operator $\mathbf{K}_{\beta}$ converges strongly to the positive-definite operator $\mathbf{K}_{0}$ as $\beta \rightarrow 0$, we can't say whether or not $\mathbf{K}_{\beta}, \beta>0$, has negative eigenvalues.

6.3. Suppose that the function $\Theta(x, y)$ in (2) is even, i.e $\Theta(-x,-y)=\Theta(x, y)$, $x, y \in \mathbb{R}$. Then the subspaces $H^{\mathrm{e}}$ and $H^{\circ}$ of $L^{2}(\mathbb{R})$ of even and odd functions are invariant for $\mathbf{K}=\mathbf{K}_{\beta}$. Consider restriction operators $\mathbf{K}^{\mathrm{e}}=\mathbf{K} \uparrow H^{\mathrm{e}}$ and $\mathbf{K}^{\mathrm{o}}=\mathbf{K} \uparrow H^{\mathrm{o}}$ and their positive eigenvalues $\lambda_{j}^{\mathrm{e}}$ and $\lambda_{j}^{\circ}, j=1,2, \ldots$, arranged in descending order. Remembering that the top eigenvalue of $\mathbf{K}$ is non-degenerate and its eigenfunction is positive a.e., one easily concludes that $\lambda_{1}^{\mathrm{e}}>\lambda_{1}^{0}$. Are there similar inequalities for the pairs $\lambda_{j}^{\mathrm{e}}, \lambda_{j}^{o}$ with $j>1$ ?

\section{Appendix. Boundedness of integral and pseudo-differential operators}

In this Appendix, for the reader's convenience we remind (without proofs) simple tests of boundedness for integral and pseudo-differential operators acting on $L^{2}\left(\mathbb{R}^{d}\right)$, $d \geq 1$. Consider the integral operator

$$
(K u)(\mathbf{x})=\int_{\mathbb{R}^{d}} K(\mathbf{x}, \mathbf{y}) u(\mathbf{y}) d \mathbf{y},
$$

with the kernel $K(\mathbf{x}, \mathbf{y})$, and the pseudo-differential operator

$$
(\mathrm{Op}(a) u)(\mathbf{x})=\frac{1}{(2 \pi)^{d}} \int_{\mathbb{R}^{d}} \int_{\mathbb{R}^{d}} e^{i(\mathbf{x}-\mathbf{y}) \cdot \xi} a(\mathbf{x}, \xi) u(\mathbf{y}) d \mathbf{y} \xi
$$

with the symbol $a(\mathbf{x}, \xi)$.

The following classical result is known as the Schur Test and it can be found, even in a more general form, in [4], Theorem 5.2.

Proposition 15. Suppose that the kernel $K$ satisfies the conditions

$$
M_{1}=\sup _{\mathbf{x}} \int_{\mathbb{R}^{d}}|K(\mathbf{x}, \mathbf{y})| d \mathbf{y}<\infty, \quad M_{2}=\sup _{\mathbf{y}} \int_{\mathbb{R}^{d}}|K(\mathbf{x}, \mathbf{y})| d \mathbf{x}<\infty .
$$

Then the operator (36) is bounded on $L^{2}\left(\mathbb{R}^{d}\right)$ and $\|K\| \leq \sqrt{M_{1} M_{2}}$.

For pseudo-differential operators on $L^{2}\left(\mathbb{R}^{d}\right)$ we use the test of boundedness found by H. O. Cordes in [2], Theorem $B_{1}^{\prime}$.

Proposition 16. Let $a(\mathbf{x}, \boldsymbol{\xi}), \mathbf{x}, \boldsymbol{\xi} \in \mathbb{R}^{d}, d \geq 1$, be a function such that its distributional derivatives of the form $\nabla_{\mathbf{x}}^{n} \nabla_{\boldsymbol{\xi}}^{m}$ a are $L^{\infty}$-functions for all $0 \leq n, m \leq r$, where

$$
r=\left[\frac{d}{2}\right]+1
$$


Then the operator (37) is bounded on $L^{2}\left(\mathbb{R}^{d}\right)$ and

$$
\|\mathrm{Op}(a)\| \leq C \max _{0 \leq n, m \leq r}\left\|\nabla_{\mathbf{x}}^{n} \nabla_{\xi}^{m} a\right\|_{L^{\infty}}
$$

with a constant $C$ depending only on $d$.

It is important for us that for $d=1$ the above test requires the boundedness of derivatives $\partial_{x}^{n} \partial_{\xi}^{m} a$ with $n, m \in\{0,1\}$ only. This result is extended to arbitrary dimensions by M. Ruzhansky and M. Sugimoto, see [13], Corollary 2.4. Recall that the classical Calderón-Vaillancourt theorem needs more derivatives with respect to each variable, see [2] and [13] for discussion. A short prove of Proposition 16 was given by I. L. Hwang in [5], Theorem 2 (see also [8], Lemma 2.3.2 for a somewhat simplified version).

\section{References}

[1] J. Adduci, Perturbations of self-adjoint operators with discrete spectrum. Ohio State University, Columbus (Ohio), 2011, Ph.D. Thesis.

[2] H. O. Cordes, On compactness of commutators of multiplications and convolutions, and boundedness of pseudodifferential operators. J. Funct. Anal. 18 (1975), 115-131. MR 0377599 Zbl 0306.47024

[3] E. B. Davies, Linear operators and their spectra. Cambridge University Press, Cambridge (U.K.), 2007. MR 2359869 Zbl 1138.47001

[4] P. R. Halmos and V. Sh. Sunder, Bounded integral operators on $L^{2}$ spaces. Springer Verlag, Berlin, 1978. MR 0517709 Zbl 0389.47001

[5] I. L. Hwang, The $L_{2}$-boundedness of pseudodifferential operators. Trans. Am. Math. Soc. 302 (1987), 55-76. MR 0887496 Zbl 0651.35089

[6] P. Krotkov and A. Chubukov, Non-Fermi liquid and pairing in electron-doped cuprates. Phys. Rev. Lett. 96 (2006), 107002-107005.

[7] P. Krotkov and A. Chubukov, Theory of non-Fermi liquid and pairing in electron-doped cuprates. Phys. Rev. B 74 (2006), 14509-14524.

[8] N. Lerner, Some facts about the Wick calculus. Pseudo-differential operators. In L. Rodino (ed.) et al., Pseudo-differential operators. Quantization and signals. Lectures given at the C.I.M.E. summer school, Cetraro, Italy, June 19-24, 2006. Springer Verlag, Berlin, 2008, 135-174. MR 2477145 Zbl 1180.35596

[9] B. Mityagin, An anisotropic integral operator in high temperature superconductivity. Isr. J. Math 181 (2011), 1-28. MR 2773035 Zbl 1217.47089

[10] M. Reed and B. Simon, Methods of modern mathematical physics I. Functional analysis and enl. ed. Academic Press, New York etc., 1980. MR 0751959 Zbl 0459.46001

[11] M. Reed and B. Simon, Methods of modern mathematical physics II. Fourier analysis, self- adjointness. Academic Press, New York etc., 1975. MR 0493420 Zbl 0308.47002 
[12] M. Reed and B. Simon, Methods of modern mathematical physics IV. Analysis of operators. Academic Press, New York etc., 1978. MR 0493421 Zbl 0401.47001

[13] M. Ruzhansky and M. Sugimoto, Global $L^{2}$-boundedness theorems for a class of Fourier integral operators. Commun. Partial Differ. Equations 31 (2006), 547-569. MR 2233032 Zbl 1106.35158

[14] M. A. Shubin, Pseudodifferential Operators and Spectral Theory. Springer Verlag, Berlin, 2001. MR 1852334 Zbl 0980.35180

[15] H. Widom, Extreme eigenvalues of translation kernels. Trans. Amer. Math. Soc. 100 (1961), 252-262. MR 0138980 Zbl 0197.10903

Received June 1, 2011; revised July 15, 2011

Boris S. Mityagin, Department of Mathematics, The Ohio State University, 231 West $18^{\text {th }}$ Ave, Columbus, OH 43210, U.S.A.

E-mail: mityagin.1@osu.edu

Alexander V. Sobolev, Department of Mathematics, University College London,

Gower Street, London, WC1E 6BT UK

E-mail: asobolev@math.ucl.ac.uk 